\title{
The tight upper bound for the number of matchings of tricyclic graphs
}

\author{
Ardeshir Dolati* \\ Department of Mathematics \& Computer Science \\ Shahed University \\ Tehran, PO Box: 18151-159, Iran \\ dolati@shahed.ac.ir
}

\author{
Somayyeh Golalizadeh \\ Young researchers club \\ Islamic Azad University \\ Ardabil branch, Ardabil, Iran \\ s.golalizadeh@gmail.com
}

Submitted: Sep 6, 2011; Accepted: Mar 23, 2012; Published: Mar 31, 2012

Mathematics Subject Classifications: 05C70, 05C90

\begin{abstract}
In this paper, we determine the tight upper bound for the number of matchings of connected $n$-vertex tricyclic graphs. We show that this bound is $13 f_{n-4}+16 f_{n-5}$, where $f_{n}$ be the $n$th Fibonacci number. We also characterize the $n$-vertex simple connected tricyclic graph for which the bound is best possible.
\end{abstract}

Keywords: Matching; Fibonacci number; Hosoya index; tricyclic graph; connected graph

\section{Introduction and preliminaries}

A matching of a graph $G=(V, E)$ is a subset $M \subseteq E$ with the property that no two different edges of $M$ share a common vertex. By $m(G, k)$ we mean the number of its $k$-matchings; matchings consisting of $k$ edges. If $n$ is the number of the vertices of $G$, then $m(G, k)=0$ when $k>\lfloor n / 2\rfloor$. Conveniently, we set $m(G, 0)=1$. The total number of matchings of $G$ is denoted by $z(G)$ and $z(G)=\sum_{k=0}^{\lfloor n / 2\rfloor} m(G, k)$. It was introduced by Hosoya [10] in 1971. He called it z-index. Later, it was renamed into Hosoya index. In this paper we refer to this invariant as $z$-index. It is a prominent example of topological indices which are of interest in combinatorial chemistry. It has applications in studies related to physico-chemical properties such as boiling point, entropy [17], and vaporization temperature. It has also a close relationship with the total $\pi$-electron energy [8].

Let $G=(V(G), E(G))$ be a simple connected graph with the vertex set $V(G)$ and the edge set $E(G)$. If $u$ and $v$ are two adjacent vertices of the graph the edge connecting

\footnotetext{
*Corresponding author: dolati@shahed.ac.ir (A. Dolati)
} 
them is denoted by $u v$. Let $v$ be a vertex of the graph, $N_{G}(v)=\{u \mid u v \in E(G)\}$ denotes the neighbors of $v$, and $d_{G}(v)=\left|N_{G}(v)\right|$ is the degree of $v$ in $G$. An end-vertex is a vertex of degree one. An end-edge is an edge incident with an end-vertex. A path consisting of exactly one end-vertex is called an end-path. A connected tricyclic graph is a simple connected graph with $n$ vertices and $n+2$ edges, for some $n \geqslant 4$. It is easy to check that, there is no $n$-vertex tricyclic graph for $n<4$. Let $x$ and $y$ be two distinct vertices of graph, an $x$-y-path is a path starting from $x$ and ending in $y$. Let $F \subseteq E(G)$, we denote by $G-F$ the subgraph of $G$ obtained from deleting the edges in $F$. If $F=\{e\}$ is a singleton set, we use $G-e$ instead of $G-\{e\}$. If $W \subseteq V(G), G-W$ denotes the subgraph of $G$ obtained from deleting the vertices in $W$. If $W=\{v\}$ is a singleton set, we use $G-v$ instead of $G-\{v\}$. Let $G=(V(G), E(G))$ and $G^{\prime}=\left(V\left(G^{\prime}\right), E\left(G^{\prime}\right)\right)$ be two graphs such that $V(G) \cap V\left(G^{\prime}\right)=\emptyset$. Suppose that $v_{1}, v_{2}, \cdots, v_{k} \in V(G)$ and $v_{1}^{\prime}, v_{2}^{\prime}, \cdots, v_{k}^{\prime} \in V\left(G^{\prime}\right)$ $(k \geqslant 1)$; by $G \triangleright v_{1}=v_{1}^{\prime}, v_{2}=v_{2}^{\prime}, \cdots, v_{k}=v_{k}^{\prime} \triangleleft G^{\prime}$ we mean the obtained graph from identifying $v_{i}$ with $v_{i}^{\prime}$ for all $i=1,2, \cdots, k$. Suppose that $P_{n}$ denotes the path on $n$ vertices, $C_{n}$ is the cycle on $n$ vertices and $S_{n}$ is the star consisting of one vertex adjacent to $n-1$ end-vertices. Amongst all $n$-vertex trees, the path $P_{n}$ has the greatest $z$-index and the star $S_{n}$ has the smallest $z$-index. This fact was established a long time ago $[8,9]$, that is, for any tree $T$ with $n$ vertices, $n=z\left(S_{n}\right) \leqslant z(T) \leqslant z\left(P_{n}\right)=f_{n+1}$, where $f_{n}$ is the $n$th Fibonacci number. Recall that the Fibonacci numbers are defined by $f_{0}=0, f_{1}=1$, and $f_{n}=f_{n-1}+f_{n-2}$ for $n \geqslant 2$. From now on we take $f_{i}$ as the $i$ th Fibonacci number. We use the following results throughout the paper.

Lemma 1. [10] If $v$ is a vertex and $e=u v$ is an edge of $G$, then

$$
\begin{gathered}
z(G)=z(G-e)+z(G-\{u, v\}), \\
z(G)=z(G-v)+\sum_{x \in N_{G}(v)} z(G-\{v, x\}) .
\end{gathered}
$$

Lemma 2. [9] If $G$ is a graph with components $G_{1}, G_{2}, G_{3}, \ldots, G_{k} k \geqslant 1$ then $z(G)=\prod_{i=1}^{k} z\left(G_{i}\right)$.

Recently, many researchers have offered various results about extremal problems in computing the total number of matchings ( $z$-index) or the total number of independent sets (Merrifield-Simmons index) for some classes of graphs. For example trees [8, 9, 15, 21], trees with fixed number of leaves [19, 24], trees with fixed diameters [16], trees with fixed maximum degrees [21], quasi-trees [12], unicyclic graphs [11, 14, 18, 20, 23], bicyclic graphs $[1,2,3,4]$, generalized-theta-graphs [6], and tricyclic graphs $[5,7,13]$ are some special classes of graphs that have been worked on. For more information, [22] is a nice survey paper on the topics. In this paper, we show that the tight upper bound of $z$-index of $n$-vertex tricyclic graphs is $13 f_{n-4}+16 f_{n-5}$. We also characterize the tricyclic $n$-vertex graph(graphs) with the largest $z$-index. The rest of the paper is organized as follows. In Section 2, we present some useful results about the Fibonacci numbers by which we offer some transformations for increasing the $z$-index. The main results are presented in Section 3, the sharp upper bound of $z$-index for all $n$-vertex tricyclic graphs is determined in Section 4, and the extremal graphs are also characterized in the same section. 


\section{Some properties of Fibonacci numbers}

The following lemmas state some facts about the Fibonacci numbers. They will be used later in our main results.

Lemma 3. Let $n$ be an integer number

1. If $n \geqslant 5$ then $f_{1} f_{n-1}>f_{3} f_{n-3}$.

2. If $n \geqslant 6$ then $f_{3} f_{n-3}>f_{i} f_{n-i}$ for $2 \leqslant i \leqslant\lfloor n / 2\rfloor$ and $i \neq 3$.

Proof. Obviously, $f_{1} f_{n-1}-f_{3} f_{n-3}=f_{n-4}$, therefore, $f_{1} f_{n-1}>f_{3} f_{n-3}$ for $n \geqslant 5$. It proves the first part.

Since $f_{3} f_{n-3}-f_{2} f_{n-2}=f_{n-5}>0$ for $n \geqslant 6$, it follows that the second part holds for all $n \geqslant 6$ if $i=2$. It will thus be sufficient to prove the second part for all $n \geqslant 8$ and $4 \leqslant i \leqslant\lfloor n / 2\rfloor$. First, note that it is rather easy to check the following:

$$
f_{i-1} f_{n-i+1}-f_{i} f_{n-i}=(-1)^{i} f_{n-2 i+1} ; \quad \text { for } i \geqslant 1 \text { and } n \geqslant 2 i-1 .
$$

We now complete the proof by showing $f_{3} f_{n-3}-f_{i} f_{n-i}>0$ as follows.

$$
\begin{aligned}
f_{3} f_{n-3}-f_{i} f_{n-i}= & \left(f_{3} f_{n-3}-f_{4} f_{n-4}\right) \\
& +\left(f_{4} f_{n-4}-f_{5} f_{n-5}\right)+\cdots \\
& +\left(f_{i-1} f_{n-i+1}-f_{i} f_{n-i}\right) .
\end{aligned}
$$

Applying (1) we deduce that

$$
\begin{aligned}
f_{3} f_{n-3}-f_{i} f_{n-i}= & f_{n-7}-f_{n-9}+f_{n-11} \\
& -f_{n-13}+\cdots(-1)^{i} f_{n-2 i+1} . \\
= & \left(f_{n-7}-f_{n-9}\right) \\
& +\left(f_{n-11}-f_{n-13}\right)+\cdots(-1)^{i} f_{n-2 i+1} \\
> & 0 .
\end{aligned}
$$

Therefore, $f_{3} f_{n-3}>f_{i} f_{n-i}$, which is the desired conclusion.

Lemma 4. [3] For $1 \leqslant k \leqslant n$, we have

$$
f_{n}=f_{k} f_{n-k+1}+f_{k-1} f_{n-k} .
$$

Let $a, b$, and $c$ be three real numbers. Simply, there are exactly 13 different arrangements according to their values. These arrangements are as follows. $a>b>c$, $a>c>b, b>a>c, b>c>a, c>a>b, c>b>a, a=b>c, c>a=b$, $a=c>b, b>a=c, a>b=c, b=c>a$, and $a=b=c$. Let $n$ be a specific integer number, for every pair of integer numbers $m, l \geqslant 2$ with $m+l=n$ we define $h(m, l)=a f_{m-1} f_{l-1}+b f_{m-2} f_{l-1}+c f_{m-1} f_{l-2}$. By the following theorem, we find a pair $(m, l)$ in which $h$ takes its maximum for every arrangement of $a, b$ and $c$. 
Theorem 5. Let $a, b$ and $c$ be three real numbers. Assume that $n \geqslant 7$ is a specific integer number. Define $h(m, l)=a f_{m-1} f_{l-1}+b f_{m-2} f_{l-1}+c f_{m-1} f_{l-2}$ for every pair of integer numbers $m, l \geqslant 2$ satisfying $m+l=n$.

According to the values of $a, b$ and $c$

Case 1) if $a>b>c$, then $h(n-2,2)>h(n-4,4) \geqslant h(m, l)$; equality holds if and only if $l=4$.

Case 2) If $a>c>b$, then $h(2, n-2)>h(4, n-4) \geqslant h(m, l)$; equality holds if and only if $m=4$.

Case3) If $b>a>c$, then $h(n-2,2)>h(3, n-3) \geqslant h(m, l)$; equality holds if and only if $m=3$.

Case 4) If $b>c>a$, then $h(3, n-3) \geqslant h(m, l)$; equality holds if and only if $m=3$.

Case 5) If $c>a>b$, then $h(2, n-2)>h(n-3,3) \geqslant h(m, l)$; equality holds if and only if $l=3$.

Case 6) If $c>b>a$, then $h(n-3,3) \geqslant h(m, l)$; equality holds if and only if $l=3$.

Case 7) If $a=b>c$, then $h(n-2,2)>h(n-4,4)=h(3, n-3) \geqslant h(m, l)$; equality holds if and only if $l=4$ or $m=3$.

Case 8) If $c>a=b$, then $h(2, n-2)=h(n-3,3) \geqslant h(m, l)$; equality holds if and only if $m=2$ or $l=3$.

Case 9) If $a=c>b$, then $h(2, n-2)>h(4, n-4)=h(n-3,3) \geqslant h(m, l)$; equality holds if and only if $m=4$ or $l=3$.

Case 10) If $b>a=c$, then $h(3, n-3)=h(n-2,2) \geqslant h(m, l)$; equality holds if and only if $m=3$ or $l=2$.

Case 11) If $a>b=c$, then $h(2, n-2)=h(n-2,2)>h(4, n-4)=h(n-4,4) \geqslant$ $h(m, l)$; equality holds if and only if $m=4$ or $l=4$.

Case 12) If $b=c>a$, then $h(3, n-3)=h(n-3,3) \geqslant h(m, l)$; equality holds if and only if $m=3$ or $l=3$.

Case 13) If $a=b=c$, then $h(m, l)$ is constant for all $m$ and $l$.

Proof. Proof of Case 1). We first prove this case for $l \geqslant 3$.

$$
\begin{aligned}
h(m, l)= & a f_{m-1} f_{l-1}+b f_{m-2} f_{l-2} \\
& +b f_{m-2} f_{l-3}+c f_{m-1} f_{l-2} \\
= & (a-b) f_{m-1} f_{l-1}+b f_{m+l-3} \\
& +(b-c) f_{m-2} f_{l-3}+c f_{m+l-4} \\
= & (a-b) f_{m-1} f_{l-1}+b f_{n-3} \\
& +(b-c) f_{m-2} f_{l-3}+c f_{n-4} .
\end{aligned}
$$

According to Lemma 3 the above expression is maximum, if and only if $l-3=1$ (i.e. $l=4)$. 
Now, suppose that $l=2$.

$$
\begin{aligned}
h(n-2,2)-h(n-4,4)= & a f_{n-3}+b f_{n-4} \\
& -2 a f_{n-5}-2 b f_{n-6}-c f_{n-5} \\
= & a f_{n-6}+b f_{n-7}-c f_{n-5} \\
> & c f_{n-6}+c f_{n-7}-c f_{n-5}=0,
\end{aligned}
$$

it follows that, $h(n-2,2)>h(n-4,4)$, and this completes the proof for Case 1 . Proof of Case 2). Argument similar to that in the proof of Case 1 proves this case. Proof of Case 3). We first prove the case for $l \geqslant 3$.

$$
\begin{aligned}
h(m, l)= & a f_{m-1} f_{l-1}+b f_{m-2} f_{l-2} \\
& +b f_{m-2} f_{l-3}+c f_{m-1} f_{l-2} \\
= & a f_{m+l-3}+(b-a) f_{m-2} f_{l-2} \\
& +(b-c) f_{m-2} f_{l-3}+c f_{m+l-4} \\
= & a f_{n-3}+(b-a) f_{m-2} f_{l-2} \\
& +(b-c) f_{m-2} f_{l-3}+c f_{n-4} .
\end{aligned}
$$

According to Lemma 3 the above expression is maximum, if and only if $m-2=1$ (i.e. $m=3$ ).

Now, let us suppose that $l=2$.

$$
\begin{aligned}
h(n-2,2)-h(3, n-3)= & a f_{n-3}+b f_{n-4} \\
& -a f_{n-4}-b f_{n-4}-c f_{n-5} \\
= & a f_{n-5}-c f_{n-5} \\
= & (a-c) f_{n-5}>0,
\end{aligned}
$$

therefore, $h(n-2,2)>h(3, n-3)$, and the theorem is also proved for this case.

Proofs of Cases 4,5 and 6 follow by the same method as in the proof of Case 3. Proof of Case 7 ). To prove this case we first suppose that $l \geqslant 3$.

$$
\begin{aligned}
h(m, l)= & a f_{m-1} f_{l-1}+b f_{m-2} f_{l-2} \\
& +b f_{m-2} f_{l-3}+c f_{m-1} f_{l-2} \\
= & b f_{m+l-3}+(b-c) f_{m-2} f_{l-3}+c f_{m+l-4} \\
= & b f_{n-3}+(b-c) f_{m-2} f_{l-3}+c f_{n-4} .
\end{aligned}
$$

According to Lemma 3 the above expression takes its maximum, if and only if $m-2=1$ (i.e. $m=3$ ) or $l-3=1$ (i.e. $l=4$ ).

We can conclude that $h(n-2,2)>h(n-4,4)=h(3, n-3)$ for $l=2$, similar to that in the proof of Case 3. 
Proof of Case 8). First, suppose that $l \geqslant 3$.

$$
\begin{aligned}
h(m, l)= & a f_{m-1} f_{l-1}+b f_{m-2} f_{l-2} \\
& +b f_{m-2} f_{l-3}+c f_{m-1} f_{l-2} \\
= & b f_{m+l-3}+b f_{m+l-4}+(c-b) f_{m-1} f_{l-2} \\
= & b f_{m+l-2}+(c-b) f_{m-1} f_{l-2} \\
= & b f_{n-2}+(c-b) f_{m-1} f_{l-2} .
\end{aligned}
$$

According to Lemma 3 the above expression is maximum, if and only if $m-1=1$ (i.e. $m=2$ ) or $l-2=1$ (i.e. $l=3$ ).

Now, suppose that $l=2$.

$$
\begin{aligned}
h(n-2,2)-h(2, n-2)= & a f_{n-3}+b f_{n-4} \\
& -a f_{n-3}-c f_{n-4} \\
= & (b-c) f_{n-4}<0,
\end{aligned}
$$

it follows that, $h(2, n-2)=h(n-3,3)>h(n-2,2)$.

Proof of Case 9). The proof of this case is similar to the proof of Case 7.

Proof of Case 10). The proof of this case can be done similar to the proof of Case 8.

Proof of Case 11). We first suppose that $l \geqslant 3$.

$$
\begin{aligned}
h(m, l)= & a f_{m-1} f_{l-1}+b f_{m-2} f_{l-2} \\
& +b f_{m-2} f_{l-3}+c f_{m-1} f_{l-2} \\
= & (a-b) f_{m-1} f_{l-1}+b f_{m+l-3}+b f_{m+l-4} \\
= & (a-b) f_{m-1} f_{l-1}+b f_{m+l-2} \\
= & (a-b) f_{m-1} f_{l-1}+b f_{n-2} .
\end{aligned}
$$

According to Lemma 3 the above expression takes its maximum, if and only if $m-1=1$ (i.e. $m=2$ ).

Now, suppose that $l=2$.

$$
\begin{aligned}
h(2, n-2)-h(n-2,2)= & a f_{n-3}+c f_{n-4} \\
& -a f_{n-3}-b f_{n-4}=0,
\end{aligned}
$$

it follows that, $h(2, n-2)=h(n-2,2)$.

Proof of Case 12). At first, suppose that $l \geqslant 3$.

$$
\begin{aligned}
h(m, l)= & a f_{m-1} f_{l-1}+b f_{m-2} f_{l-2} \\
& +b f_{m-2} f_{l-3}+c f_{m-1} f_{l-2} \\
= & a f_{m+l-3}+(b-a) f_{m-2} f_{l-2}+b f_{m+l-4} \\
= & a f_{n-3}+(b-a) f_{m-2} f_{l-2}+b f_{n-4} .
\end{aligned}
$$


According to the Lemma 3 the above expression is maximum, if and only if $m-2=1$ (i.e. $m=3$ ) or $l-2=1$ (i.e. $l=3$ ).

Now, suppose that $l=2$.

$$
\begin{aligned}
h(3, n-3)-h(n-2,2)= & a f_{n-4}+b f_{n-4}+c f_{n-5} \\
& -a f_{n-3}-b f_{n-4} \\
= & -a f_{n-5}+c f_{n-5} \\
= & (c-a) f_{n-5}>0 .
\end{aligned}
$$

Therefore $h(3, n-3)>h(n-2,2)$, which completes the proof for Case 12 .

Proof of Case (13). In this case we show that for all $m$ and $l, h(m, l)=a f_{n-2}$. First, suppose that $l \geqslant 3$.

$$
\begin{aligned}
h(m, l)= & a f_{m-1} f_{l-1}+b f_{m-2} f_{l-2} \\
& +b f_{m-2} f_{l-3}+c f_{m-1} f_{l-2} \\
& +b f_{m+l-3}+b f_{m+l-4} \\
= & b f_{m+l-2}=b f_{n-2} .
\end{aligned}
$$

Now, suppose that $l=2$.

$$
\begin{aligned}
h(n-2,2)-h(m, l) & =a f_{n-3}+b f_{n-4}-b f_{n-2} \\
& =b f_{n-2}-b f_{n-2}=0,
\end{aligned}
$$

and then the proof is completed.

\section{How can the number of matchings be increased?}

This section is devoted to the results by which one can construct a simple connected tricyclic graph with a larger $z$-index from a non-extremal one. We call them the increasing transformations. Some useful increasing transformations for $z$-index are presented here.

Transformation I. [21] Let $G$ be a non-trivial connected graph and choose $u \in V(G)$. Suppose that $H_{1}$ denotes the graph obtained from identifying $u$ with the vertex $v_{k}$ of a simple path $v_{1} v_{2} \cdots v_{n}, 1<k<n$; and $H_{2}$ is obtained from $H_{1}$ by deleting $v_{k-1} v_{k}$ and adding $v_{1} v_{n}$, then $z\left(H_{1}\right)<z\left(H_{2}\right)$ (see Fig 1 ).

Transformation II. [1] Let $P=u u_{1} u_{2} \cdots u_{t} v$ be a path in $G$, where the degrees of $u_{1}, \cdots, u_{t}$ in $G$ are 2 . Assume that $K_{1}$ denotes the graph obtained from identifying $u$ with the vertex $v_{k}$ of a simple path $v_{1} v_{2} \cdots v_{k}$ and identifying $v$ with the vertex $v_{k+1}$ of another simple path $v_{k+1} v_{k+2} \cdots v_{n}, 1<k<n$. Now suppose that $K_{2}$ is obtained from $K_{1}$ by deleting $v_{k-1} v_{k}$ and adding $v_{1} v_{n} ; K_{3}$ is obtained from $K_{1}$ by deleting $v_{k+1} v_{k+2}$ and adding $v_{1} v_{n}$, then $z\left(K_{1}\right)<z\left(K_{2}\right)$ or $z\left(K_{1}\right)<z\left(K_{3}\right)$ (see Fig 2). 


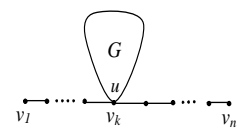

$H_{I}$

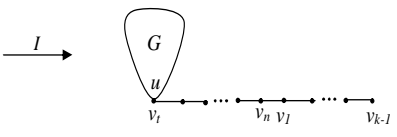

$\mathrm{H}_{2}$

Figure 1: Transformation I

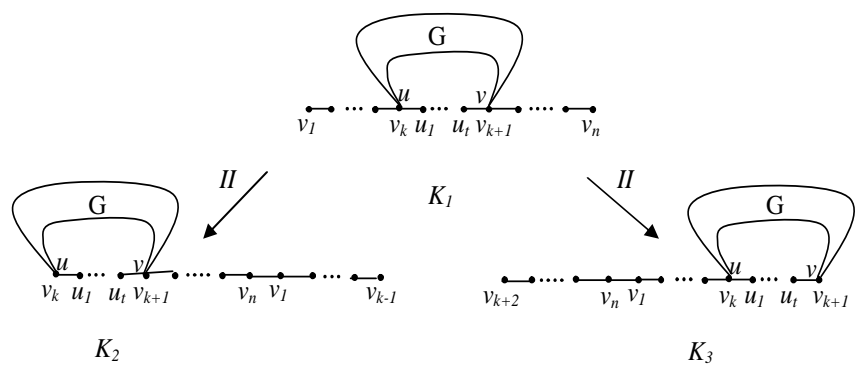

Figure 2: Transformation II

Transformation III. [1] Let $P=u_{0} u_{1} u_{2} \cdots u_{t+1}$ be a path or a cycle (if $u_{0}=u_{t+1}$ ) in $G$, where $t \geqslant 1$ and the degrees of $u_{1}, \cdots, u_{t}$ in $G$ are 2. Suppose that $F_{1}$ denotes the graph obtained from identifying $u_{r}(0 \leqslant r \leqslant t)$ with the vertex $v_{k}$ of a simple path $v_{1} v_{2} \cdots v_{k}(k \geqslant 2) ; F_{2}$ is obtained from $F_{1}$ by deleting $u_{r} u_{r+1}$ and adding $u_{r+1} v_{1}$, then $z\left(F_{1}\right)<z\left(F_{2}\right)$ (see Fig 3).

Transformation $\mathcal{A}$. Let $H \nsubseteq P_{3}$ be a simple graph, where $u$ and $v$ are two nonisolated and non-adjacent vertices of it. Suppose that $G_{s}$ denotes the graph obtained from identifying $u$ with the vertex $w_{0}$ of a cycle $w_{0} w_{1} \cdots w_{r-1} w_{0}(r \geqslant 4)$ and identifying $v$ with the vertex $w_{s}(1<s<r-1)$ of the cycle. $G_{1}$ is obtained from $G_{s}$ by deleting edges $w_{0} w_{1}$ and $w_{s} w_{s+1}$ and adding edges $w_{0} w_{s}$ and $w_{s+1} w_{1}$ (see Fig. 4).

Lemma 6. If $G_{s}$ and $G_{1}$ are the graphs introduced in Transformation $\mathcal{A}$, then $z\left(G_{s}\right)<$ $z\left(G_{1}\right)$.

Proof. Repeated application of Lemma 1 and use of Lemma 3 enable us to prove the assertion.

Although, Transformation $\mathcal{A}$ is a new increasing transformation for $z$-index, there are many increasing transformations to be presented here by the following theorem. Before stating the theorem, let us introduce a notation. Let $m \geqslant 2, n \geqslant 2$ be two integer numbers. Suppose that $u, v, w$ and $z$ are four distinct vertices of $G$. By $G_{w, z}^{u, v}(m, l)$, we denote the graph obtained from identifying the vertices $u$ and $v$ with the end-vertices of a path on $m$ vertices, and identifying the vertices $w$ and $z$ with the end-vertices of a path on $l$ vertices. 


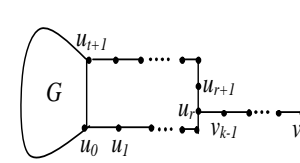

$F_{1}$

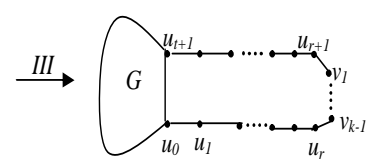

$F_{2}$

Figure 3: Transformation III

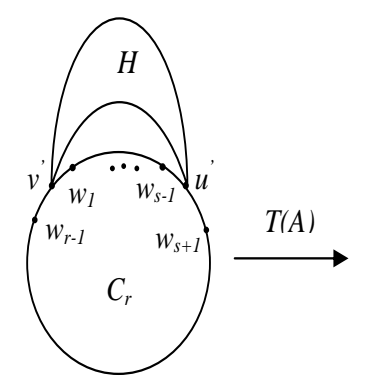

$G_{S}$

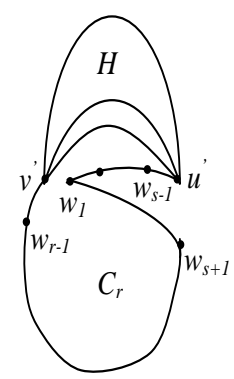

$G_{1}$

Figure 4: Illustration of Transformation $\mathcal{A}$

Theorem 7. Let $G$ be a simple graph and $u, v, w$ and $z$ be four distinct vertices of $G$. Suppose that $\alpha=z(G), \beta=z(G-u)+z(G-v), \gamma=z(G-w)+z(G-z), \delta=z(G-$ $\{u, w\})+z(G-\{v, w\})+z(G-\{u, z\})+z(G-\{v, z\}), \zeta=z(G-\{u, v\}), \eta=z(G-\{w, z\})$, $\lambda=z(G-\{u, v, w\})+z(G-\{u, v, z\}), \mu=z(G-\{u, w, z\})+z(G-\{v, w, z\})$ and $\tau=z(G-\{u, v, w, z\})$.

Suppose that $n \geqslant 6$ be an integer number and $m, l \geqslant 2$ integer numbers satisfying $m+l=n$.

Case 1) Suppose that $\alpha-\delta+\tau+\zeta+\eta>\beta-\lambda-\zeta>\gamma-\mu-\eta$.

1.a) If $w z$ is an edge of $G$, then $z\left(G_{w, z}^{u, v}(n-4,4)\right) \geqslant z\left(G_{w, z}^{u, v}(m, l)\right)$; equality holds if and only if $l=4$.

1.b) If $w z$ is not an edge of $G$, then $z\left(G_{w, z}^{u, v}(n-2,2)\right)>z\left(G_{w, z}^{u, v}(n-4,4)\right) \geqslant$ $z\left(G_{w, z}^{u, v}(m, l)\right)$; equality holds if and only if $l=4$.

Case 2) Suppose that $\alpha-\delta+\tau+\zeta+\eta>\gamma-\mu-\eta>\beta-\lambda-\zeta$.

2.a) If $u v$ is an edge of $G$, then $z\left(G_{w, z}^{u, v}(4, n-4)\right) \geqslant z\left(G_{w, z}^{u, v}(m, l)\right)$; equality holds if and only if $m=4$.

2.b) If $u v$ is not an edge of $G$, then $z\left(G_{w, z}^{u, v}(2, n-2)\right)>z\left(G_{w, z}^{u, v}(4, n-4)\right) \geqslant$ $z\left(G_{w, z}^{u, v}(m, l)\right)$; equality holds if and only if $m=4$.

Case 3) Suppose that $\beta-\lambda-\zeta>\alpha-\delta+\tau+\zeta+\eta>\gamma-\mu-\eta$.

3.a) If $w z$ is an edge of $G$, then $z\left(G_{w, z}^{u, v}(3, n-3)\right) \geqslant z\left(G_{w, z}^{u, v}(m, l)\right)$; equality holds if and only if $m=3$.

3.b) If $w z$ is not an edge of $G$, then $z\left(G_{w, z}^{u, v}(n-2,2)\right)>z\left(G_{w, z}^{u, v}(3, n-3)\right) \geqslant$ $z\left(G_{w, z}^{u, v}(m, l)\right)$; equality holds if and only if $m=3$. 
Case 4) If $\beta-\lambda-\zeta>\gamma-\mu-\eta>\alpha-\delta+\tau+\zeta+\eta$, then $z\left(G_{w, z}^{u, v}(3, n-3)\right) \geqslant z\left(G_{w, z}^{u, v}(m, l)\right)$; equality holds if and only if $m=3$.

Case 5) Suppose that $\gamma-\mu-\eta>\alpha-\delta+\tau+\zeta+\eta>\beta-\lambda-\zeta$.

5.a) If $u v$ is an edge of $G$, then $z\left(G_{w, z}^{u, v}(n-3,3)\right) \geqslant z\left(G_{w, z}^{u, v}(m, l)\right)$; equality holds if and only if $l=3$.

5.b) If uv is not an edge of $G$, then $z\left(G_{w, z}^{u, v}(2, n-2)\right)>z\left(G_{w, z}^{u, v}(n-3,3)\right) \geqslant z\left(G_{w, z}^{u, v}(m, l)\right)$; equality holds if and only if $l=3$.

Case 6) If $\gamma-\mu-\eta>\beta-\lambda-\zeta>\alpha-\delta+\tau+\zeta+\eta$, then $z\left(G_{w, z}^{u, v}(n-3,3)\right) \geqslant z\left(G_{w, z}^{u, v}(m, l)\right)$; equality holds if and only if $l=3$.

Case 7) Suppose that $\alpha-\delta+\tau+\zeta+\eta=\beta-\lambda-\zeta>\gamma-\mu-\eta$.

7.a) If $w z$ is an edge of $G$, then $z\left(G_{w, z}^{u, v}(n-4,4)\right)=z\left(G_{w, z}^{u, v}(3, n-3)\right) \geqslant z\left(G_{w, z}^{u, v}(m, l)\right)$; equality holds if and only if $l=4$ or $m=3$.

7.b) If $w z$ is not an edge of $G$, then then $z\left(G_{w, z}^{u, v}(n-2,2)\right)>z\left(G_{w, z}^{u, v}(n-4,4)\right)=$ $z\left(G_{w, z}^{u, v}(3, n-3)\right) \geqslant z\left(G_{w, z}^{u, v}(m, l)\right)$; equality holds if and only if $l=4$ or $m=3$.

Case 8) Suppose that $\alpha-\delta+\tau+\zeta+\eta=\beta-\lambda-\zeta<\gamma-\mu-\eta$.

8.a) If uv is an edge of $G$, then $z\left(G_{w, z}^{u, v}(n-3,3)\right) \geqslant z\left(G_{w, z}^{u, v}(m, l)\right)$; equality holds if and only if $l=3$.

8.b) If $u v$ is not an edge of $G$, then $z\left(G_{w, z}^{u, v}(2, n-2)\right)=z\left(G_{w, z}^{u, v}(n-3,3)\right) \geqslant$ $z\left(G_{w, z}^{u, v}(m, l)\right)$; equality holds if and only if $m=2$ or $l=3$.

Case 9) Suppose that $\alpha-\delta+\tau+\zeta+\eta=\gamma-\mu-\eta>\beta-\lambda-\zeta$.

9.a) If $u v$ is an edge of $G$, then $z\left(G_{w, z}^{u, v}(4, n-4)\right)=z\left(G_{w, z}^{u, v}(n-3,3)\right) \geqslant z\left(G_{w, z}^{u, v}(m, l)\right)$; equality holds if and only if $m=4$ or $l=3$.

9.b) If $u v$ is not an edge of $G$, then $z\left(G_{w, z}^{u, v}(2, n-2)\right)>z\left(G_{w, z}^{u, v}(4, n-4)\right)=z\left(G_{w, z}^{u, v}(n-\right.$ $3,3)) \geqslant z\left(G_{w, z}^{u, v}(m, l)\right)$; equality holds if and only if $m=4$ or $l=3$.

Case 10) Suppose that $\alpha-\delta+\tau+\zeta+\eta=\gamma-\mu-\eta<\beta-\lambda-\zeta$.

10.a) If $w z$ is an edge of $G$, then $z\left(G_{w, z}^{u, v}(3, n-3)\right) \geqslant z\left(G_{w, z}^{u, v}(m, l)\right)$; equality holds if and only if $m=3$.

10.b) If $w z$ is not an edge of $G$, then $z\left(G_{w, z}^{u, v}(3, n-3)\right)=z\left(G_{w, z}^{u, v}(n-2,2)\right) \geqslant$ $z\left(G_{w, z}^{u, v}(m, l)\right)$; equality holds if and only if $m=3$ or $l=2$.

Case 11) Suppose that $\alpha-\delta+\tau+\zeta+\eta>\beta-\lambda-\zeta=\gamma-\mu-\eta$.

11.a) If $w z$ and $u v$ are edges of $G$, then $z\left(G_{w, z}^{u, v}(4, n-4)\right)=z\left(G_{w, z}^{u, v}(n-4,4)\right) \geqslant$ $z\left(G_{w, z}^{u, v}(m, l)\right)$; equality holds if and only if $m=4$ or $l=4$.

11.b) If $w z$ and $u v$ are not edges of $G$, then $z\left(G_{w, z}^{u, v}(2, n-2)\right)=z\left(G_{w, z}^{u, v}(n-2,2)\right)>$ $z\left(G_{w, z}^{u, v}(4, n-4)\right)=z\left(G_{w, z}^{u, v}(n-4,4)\right) \geqslant z\left(G_{w, z}^{u, v}(m, l)\right)$; equality holds if and only if $m=4$ or $l=4$.

11.c) If $w z$ is an edge of $G$ and $u v$ is not edge of $G$, then $z\left(G_{w, z}^{u, v}(2, n-2)\right)=z\left(G_{w, z}^{u, v}(n-\right.$ $4,4)) \geqslant z\left(G_{w, z}^{u, v}(m, l)\right)$; equality holds if and only if $m=2$ or $l=4$.

11.d) If $u v$ is an edge of $G$ and $w z$ is not edge of $G$, then $z\left(G_{w, z}^{u, v}(4, n-4)\right)=$ $z\left(G_{w, z}^{u, v}(n-2,2)\right) \geqslant z\left(G_{w, z}^{u, v}(m, l)\right)$; equality holds if and only if $m=4$ or $l=2$.

Case 12) If $\alpha-\delta+\tau+\zeta+\eta<\beta-\lambda-\zeta=\gamma-\mu-\eta$, then $z\left(G_{w, z}^{u, v}(3, n-3)=\right.$ $z\left(G_{w, z}^{u, v}(n-3,3) \geqslant z\left(G_{w, z}^{u, v}(m, l)\right.\right.$; equality holds if and only if $m=3$ or $l=3$.

Case 13) If $\alpha-\delta+\tau+\zeta+\eta=\beta-\lambda-\zeta=\gamma-\mu-\eta$, then $z\left(G_{w, z}^{u, v}(m, l)\right)$ is constant for all $m$ and $l$. 
Proof. Suppose that the leaves of the paths $P_{m}$ and $P_{l}$ are identified with $u, v, w$ and $z$. Let $\widehat{G}=G_{w, z}^{u, v}(m, l)$. At first by recursively use of the first part of Lemma 1 and deleting the four edges of the aforementioned paths incident with the vertices $u, v, w$ and $z$, we have

$$
\begin{aligned}
z(\widehat{G})= & \alpha f_{m-1} f_{l-1}+\beta f_{m-2} f_{l-1}+\gamma f_{m-1} f_{l-2} \\
& +\delta f_{m-2} f_{l-2}+\zeta f_{m-3} f_{l-1}+\eta f_{m-1} f_{l-3} \\
& +\lambda f_{m-3} f_{l-2}+\mu f_{m-2} f_{l-3}+\tau f_{m-3} f_{l-3}
\end{aligned}
$$

By using the Lemma 4

$$
\begin{aligned}
z(\widehat{G})= & \alpha f_{m-1} f_{l-1}+(\beta-\lambda) f_{m-2} f_{l-1} \\
& +\lambda f_{m+l-4}+(\gamma-\mu) f_{m-1} f_{l-2}+\mu f_{m+l-4} \\
& +(\delta-\tau) f_{m-2} f_{l-2}+\tau f_{m+l-5} \\
& +\zeta\left(f_{m-3} f_{l-2}+f_{m-3} f_{l-3}\right) \\
& +\eta\left(f_{m-2} f_{l-3}+f_{m-3} f_{l-3}\right) \\
= & \alpha f_{m-1} f_{l-1}+(\beta-\lambda-\zeta) f_{m-2} f_{l-1} \\
& +(\lambda+\zeta) f_{m+l-4}+(\gamma-\mu-\eta) f_{m-1} f_{l-2} \\
& +(\mu+\eta) f_{m+l-4}+(\delta-\tau-\zeta-\eta) f_{m-2} f_{l-2} \\
& +(\tau+\zeta+\eta) f_{m+l-5} \\
= & (\alpha-\delta+\tau+\zeta+\eta) f_{m-1} f_{l-1} \\
& +(\beta-\lambda-\zeta) f_{m-2} f_{l-1}+(\gamma-\mu-\eta) f_{m-1} f_{l-2} \\
& +(\lambda+\zeta+\mu+\eta) f_{m+l-4}+(\tau+\zeta+\eta) f_{m+l-5} \\
& +(\delta-\tau-\zeta-\eta) f_{m+l-3} \\
= & (\alpha-\delta+\tau+\zeta+\eta) f_{m-1} f_{l-1} \\
& +(\beta-\lambda-\zeta) f_{m-2} f_{l-1}+(\gamma-\mu-\eta) f_{m-1} f_{l-2} \\
& +(\lambda+\zeta+\mu+\eta) f_{n-4}+(\tau+\zeta+\eta) f_{n-5} \\
& +(\delta-\tau-\zeta-\eta) f_{n-3} \\
= & (\alpha-\delta+\tau+\zeta+\eta) f_{m-1} f_{l-1} \\
& +(\beta-\lambda-\zeta) f_{m-2} f_{l-1}+(\gamma-\mu-\eta) f_{m-1} f_{l-2}+k
\end{aligned}
$$

where $k$ is a constant and equal to $(\lambda+\zeta+\mu+\eta) f_{n-4}+(\tau+\zeta+\eta) f_{n-5}+(\delta-\tau-\zeta-\eta) f_{n-3}$. Therefore, by Theorem 5 , the assertion is immediately proved.

Let $P_{m}=v_{1} v_{2} \cdots v_{m}(m \geqslant 2)$ be a path and $C_{l}=w_{1} w_{2} \cdots w_{l} w_{1}$ be a cycle, by $P C_{m, l}$, we mean $P_{m} \triangleright v_{m}=w_{k} \triangleleft C_{l}$ for some $k=1,2, \cdots, l$. Obviously, $P C_{m, l}$ has an unique end-vertex. Let us now state a corollary of the above theorem. We shall directly use the corollary as an increasing transformation for $z$-index. 


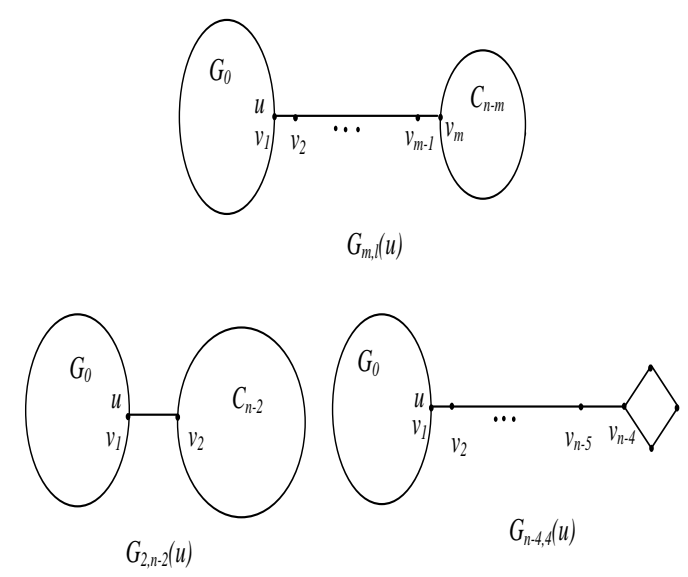

Figure 5: Illustration Corollary 8

Corollary 8. Let $u$ be a vertex of a simple graph $G_{0}$. Suppose that $m, l$ and $n$ are three integer numbers such that $m \geqslant 2, l \geqslant 3, n \geqslant 6$ and $m+l=n$. Let $G_{m, l}(u)$ denotes the graph obtained from identifying $u$ with the end-vertex of $P C_{m, l}$.

i) If $2 z\left(G_{0}-u\right)<z\left(G_{0}\right)$, then $z\left(G_{2, n-2}(u)\right) \geqslant z\left(G_{m, l}(u)\right)$ with equality holding if and only if $G \cong G_{2, n-2}(u)$.

ii) If $z\left(G_{0}\right)=2 z\left(G_{0}-u\right)$, then $z\left(G_{n-4,4}(u)\right)=z\left(G_{2, n-2}(u)\right) \geqslant z\left(G_{m, l}(u)\right)$ with equality holding if and only if $G \cong G_{n-4,4}(u)$ or $z\left(G_{2, n-2}(u)\right)$.

iii) If $z\left(G_{0}\right)<2 z\left(G_{0}-u\right)$, then $z\left(G_{n-4,4}(u)\right) \geqslant z\left(G_{m, l}(u)\right)$ with equality holding if and only if $G \cong G_{n-4,4}(u)$. See Figure 5 .

\section{Constructing the extremal graphs}

We are now in a position to construct the $n$-vertex connected tricyclic graph with the largest $z$-index. For cases $n=4, n=5$, and $n=6$ there are a few $n$-vertex tricyclic graphs. Therefore, we can determine the extremal ones for these cases by a direct comparison of their $z$-indices. The extremal graphs and their $z$-indices are depicted in Fig. 9. It will thus be sufficient to determine the extremal $n$-vertex tricyclic graph for every $n \geqslant 7$. Let us first consider some notations and definitions. We call a tricyclic graph an elementary tricyclic graph if it has no end-vertex. A path is called a $k$-path if all its internal vertices are of degree $k$. If $G$ is an elementary tricyclic graph, its skeleton is a multiple graph obtained by replacing every maximal 2-path by an edge. We denote the skeleton of $G$ by $\mathcal{S}(G)$

Definition 9. Let $G_{1}$ and $G_{2}$ be two elementary tricyclic graphs. We say $G_{1}$ and $G_{2}$ are related with respect to $\sim$ and write $G_{1} \sim G_{2}$ if their skeleton are isomorphic.

Relation $\sim$ is easily seen to be an equivalence relation. For $n \geqslant 7$ the set of $n$-vertex elementary tricyclic graphs consists of 15 distinct equivalence classes [7]. These 15 classes 


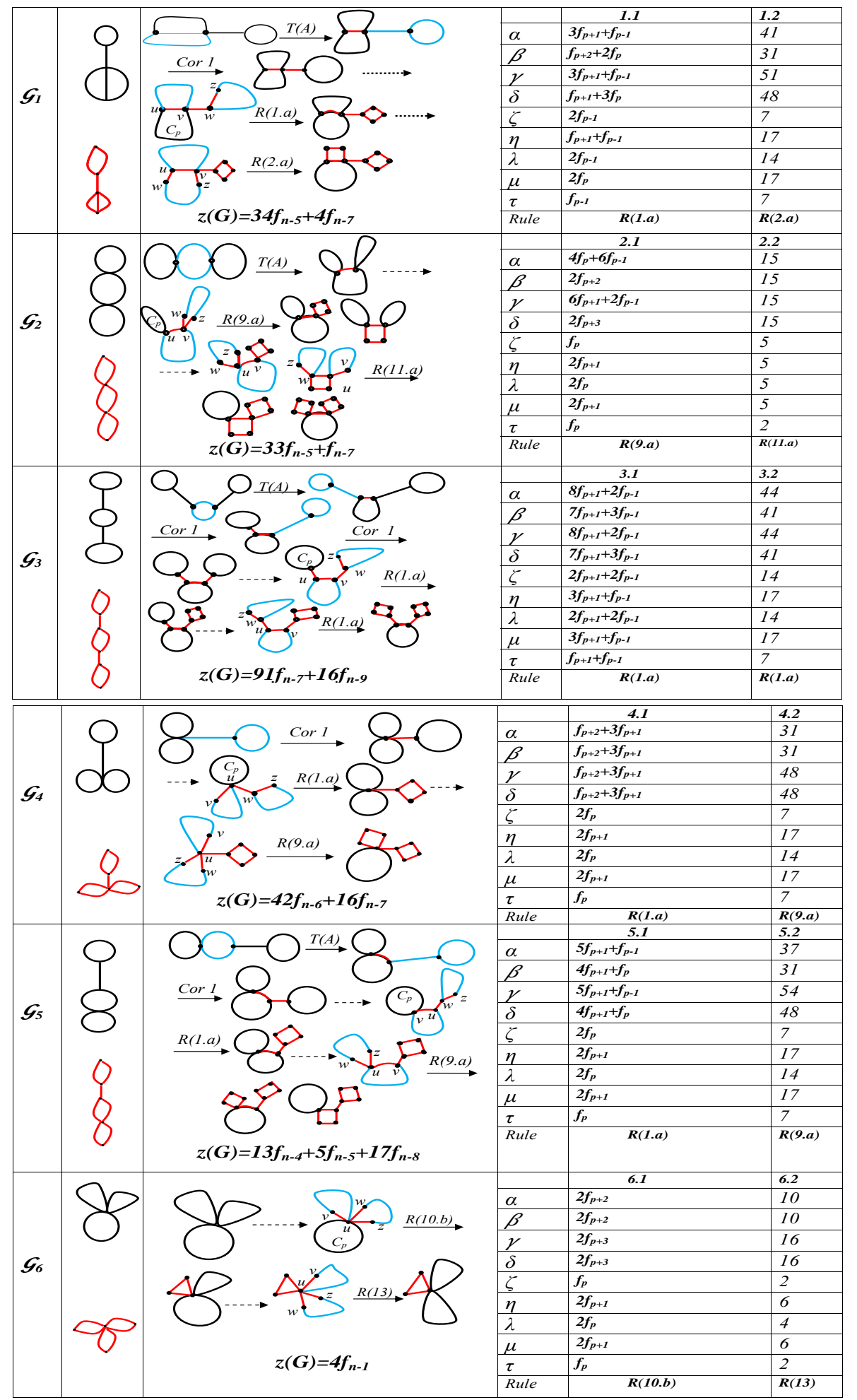

Figure 6: Classes 1-6, the length of the paths depicted in red are one. The selected cycle, $P C_{m, l}$, and pair of 2-paths for applying Transformation $\mathcal{A}$, Corollary 8 , and increasing rules of Theorem 12, respectively, are depicted in blue. 


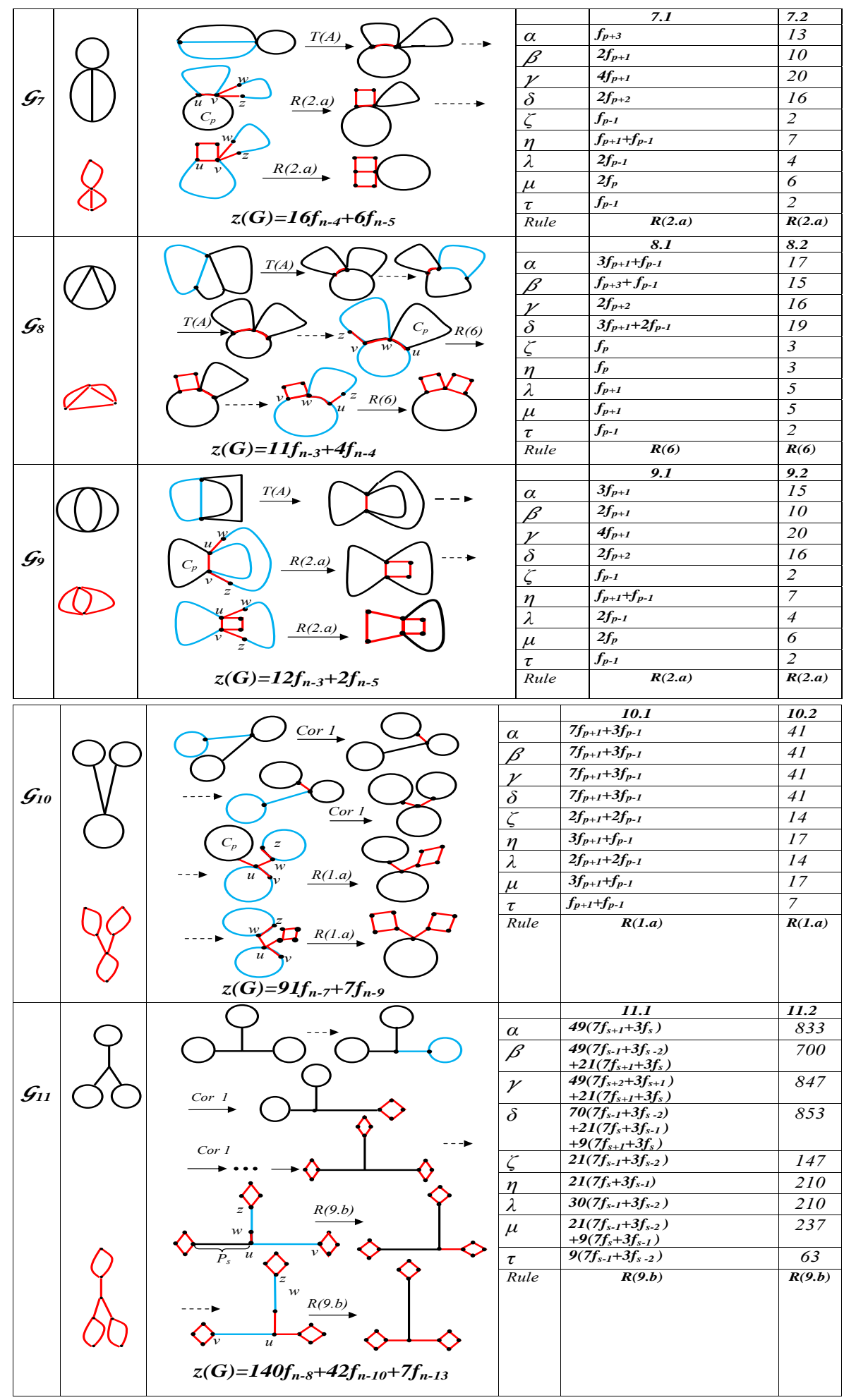

Figure 7: Classes 7-11, the length of the paths depicted in red are one. The selected cycle, $P C_{m, l}$, and pair of 2-paths for applying Transformation $\mathcal{A}$, Corollary 8, and increasing rules of Theorem 12, respectively, are depicted in blue. 


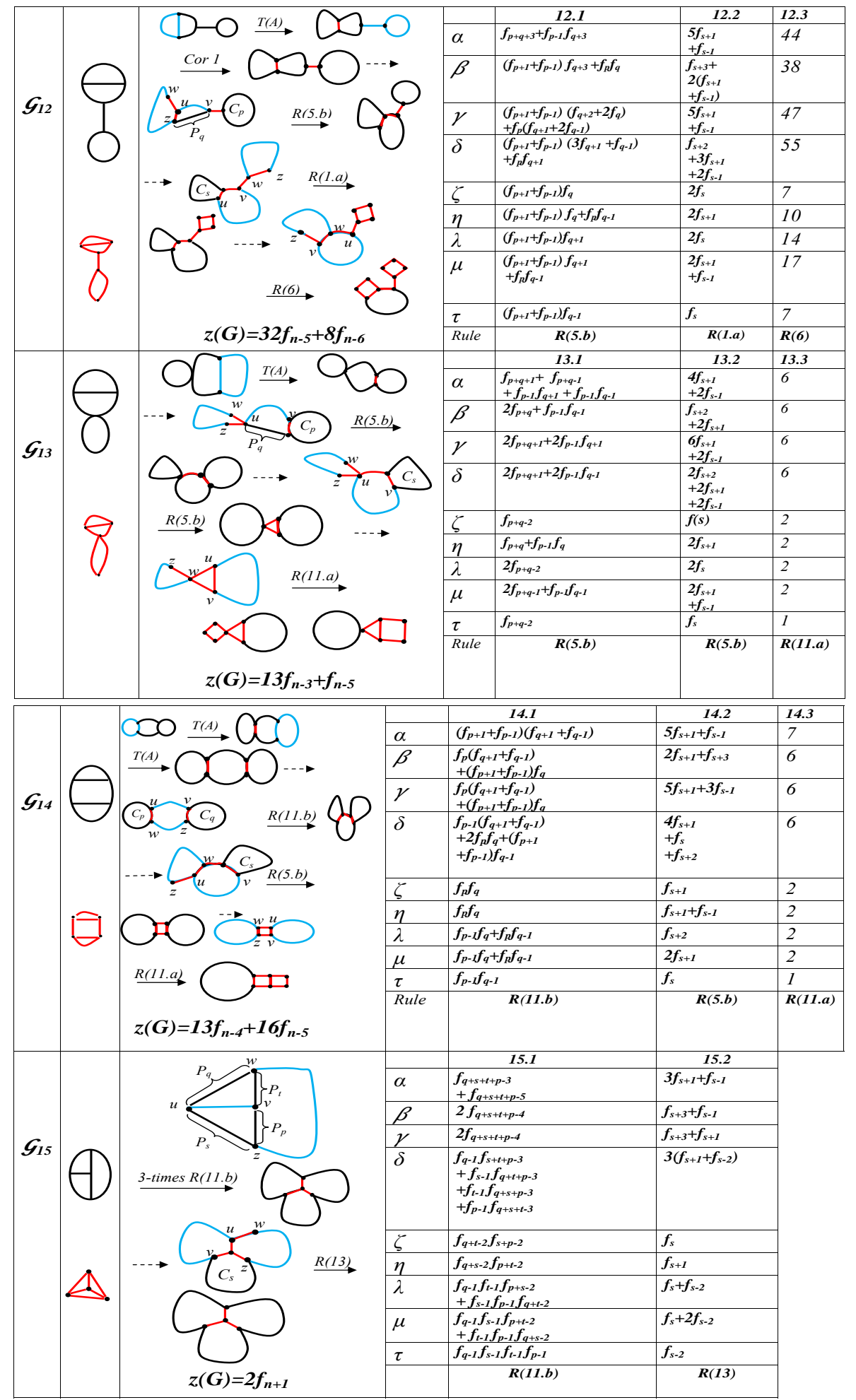

Figure 8: Classes 12-15, the length of the paths depicted in red are one. The selected cycle, $P C_{m, l}$, and pair of 2-paths for applying Transformation $\mathcal{A}$, Corollary 8, and increasing rules of Theorem 12, respectively, are depicted in blue. 
and their skeletons are depicted in the second columns of the tables in Figures 6, 7, and 8; the skeleton of each class is depicted in red. We denote these classes by $\mathcal{G}_{1}, \mathcal{G}_{2}, \cdots, \mathcal{G}_{15}$. In each of them, the length of the 2-paths can be changed without changing the class. On the other hand, repeating Transformations $1-3$, changes any non-elementary tricyclic graph into an elementary tricyclic graph and increases the $z$-index. Thus, to find the $n$-vertex connected tricyclic graph with the largest $z$-index, we only need to find the extremal graph(s) in each class. In the remainder of the section, we construct the extremal graph or extremal graphs of each of classes $\mathcal{G}_{1}, \mathcal{G}_{2}, \cdots, \mathcal{G}_{15}$, by using Transformation $\mathcal{A}$ and the rules characterized in Theorem 7 . Finally, we characterize the $n$-vertex connected tricyclic graph with the largest $z$-index, by a direct comparison of the $z$-indices of the extremal graphs.

Remark 10. Transformation $\mathcal{A}$ can easily be shown to be a special case of the rules characterized in Theorem 7.

Remark 11. Changing the order of the application of rules of Theorem 7 does not change the results.

Note that each graph in $\mathcal{G}_{i}$ can be characterized by replacing some edges of the skeleton by 2-paths of specified length. We call the lengths of these 2-paths the "decision parameters" to maximize $z$-index of the graph. We are left with the task of properly determining these lengths for constructing the extremal graph in every class $\mathcal{G}_{i}$. For this purpose, we do one of the following tasks in every stage:

- We select a proper cycle of graph for doing Transformation $\mathcal{A}$, if there is any. Each of these cycles selected for this task, is depicted blue in the graphs of the third column of tables in Figures 6, 7, and 8.

- We select a proper $P C_{m, l}$ of graph for doing Corollary 8, if there is any. Each of these $P C_{m, l}$ selected for this task, is depicted blue in the graph of the third column of tables in Figures 6, 7, and 8.

- We consider two disjoint 2-paths $P_{1}$ and $P_{2}$ whose end vertices are four distinct vertices $u, v, w$, and $z$. Each pair of these 2-paths selected for this task, are depicted blue in the graph of the third column of tables in Figures 6, 7, and 8. According to the vertices $u, v, w$, and $z$ we compute the parameters $\alpha, \beta, \gamma, \delta, \zeta, \eta, \lambda, \mu$, and $\tau$ of Theorem 7 by which we select the corresponding increasing rule implied by the theorem. The values of these parameters by which we select the rules are depicted in the fifth and subsequent columns. Using the transformation rule redetermines the new lengths of these paths such that the $z$-index of the new graph becomes the largest value without affecting the sum of their lengths.

Repeating the procedure above determines the extremal graph in each class. For example, in class $\mathcal{G}_{1}$, we may select a cycle and use Transformation $\mathcal{A}$. Then we may select a $P C_{m, l}$ and use Corollary 8. Finally, we may select two pairs of 2-paths and for each compute the values of parameters $\alpha, \beta, \gamma, \delta, \zeta, \eta, \lambda, \mu$, and $\tau$ of Theorem 7 , then the corresponding increasing rules will be determined and applied (see fifth and sixth Columns 


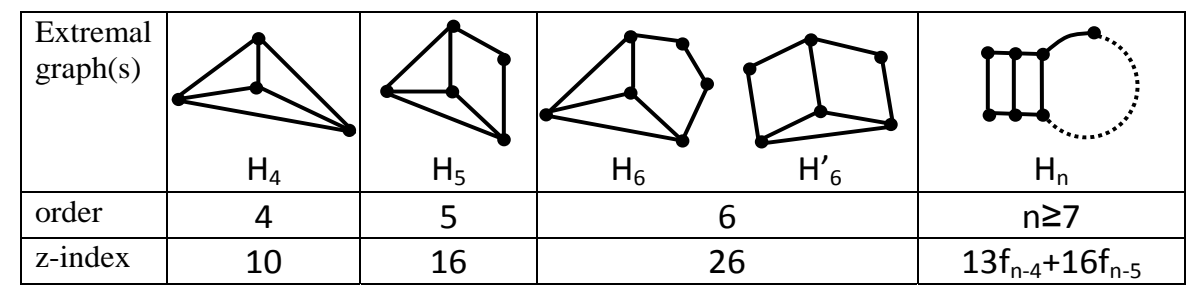

Figure 9: The extremal n-vertex tricyclic graphs

of the corresponding row of the table of Fig 6). The details of the calculations are as follow. For the first pair of 2-paths : $\alpha=3 f_{p+1}+f_{p-1}, \beta=f_{p+2}+2 f_{p}, \gamma=3 f_{p+1}+f_{p-1}$, $\delta=f_{p+1}+3 f_{p}, \zeta=2 f_{p-1}, \eta=f_{p+1}+f_{p-1}, \lambda=2 f_{p-1}, \mu=2 f_{p}, \tau=f_{p-1}$. Since $\alpha-\delta+\tau+\zeta+\eta>\beta-\lambda-\zeta>\gamma-\mu-\eta$ and $w z$ is an edge, the corresponding increasing rule is $\mathbf{R}(\mathbf{1} \cdot \mathbf{a})$.

For the second pair of 2-paths: $\alpha=41, \beta=31, \gamma=51, \delta=48, \zeta=7, \eta=17, \lambda=14$, $\mu=17, \tau=7$. Since $\alpha-\delta+\tau+\zeta+\eta>\gamma-\mu-\eta>\beta-\lambda-\zeta$ and $w z$ is not an edge, the corresponding increasing rule is $\mathbf{R}(\mathbf{2} \cdot \mathbf{a})$.

After constructing the extremal graph of each class, the sharp upper bound of $z$ index of $n$-vertex tricyclic graphs can be determined by comparing the $z$-indices of these constructed extremal graphs. Therefore, the extremal graph can also be characterized. These results are summarized in the following theorem.

Theorem 12. Let $G$ be an arbitrary n-vertex connected tricyclic graph.

If $n=4$ then $z(G) \leqslant 10$ with equality holding if and only if $G \cong H_{4}$.

If $n=5$ then $z(G) \leqslant 16$ with equality holding if and only if $G \cong H_{5}$.

If $n=6$ then $z(G) \leqslant 26$ with equality holding if and only if $G \cong H_{6}$ or $G \cong H_{6}^{\prime}$.

If $n \geqslant 7 z(G) \leqslant 13 f_{n-4}+16 f_{n-5}$ with equality holding if and only if $G \cong H_{n}$, see Fig. 9 .

\section{References}

[1] H. Deng, The largest Hosoya index of ( $\mathrm{n}, \mathrm{n}+1)$-graphs, Comput. Math. Appl., 56(10):2499-2506, 2008.

[2] H. Deng, The smallest Hosoya index in (n, n+1)-graphs, J. Math. Chem., 43(1):119$133,2008$.

[3] H. Deng, The smallest Merrifield-Simmons index of (n, n+1)-graphs, Math. Comput. Modelling, 49(1-2):320-326, 2009.

[4] H. Deng, S. Chen and J. Zhang, The Merrifield-Simmons index in (n, n+1)-graphs, J. Math. Chem., 43(1):75-91, 2008.

[5] A. Dolati, On the number of independent sets in cycle-separated tricyclic graphs, Comput. Math. Appl., 61(6):1542-1546, 2011.

[6] A. Dolati and S. Golalizadeh, Sharp bounds for the number of matchings in generalized-theta-graphs, Discussiones Mathematicae Graph Theory, to appear. 
[7] A. Dolati, M. Haghighat, S. Golalizadeh and M. Safari, The smallest Hosoya index of connected tricyclic graphs, MATCH Commun. Math. Comput. Chem., 65:57-70, 2011.

[8] I. Gutman, Acyclic systems with extremal Hückel $\pi$-electron energy, Theor. Chim. Acta, 45:79-87, 1977.

[9] I. Gutman and O. E. Polansky, Mathematical Concepts in Organic Chemistry. Springer-Verlag, Berlin, 1986.

[10] H. Hosoya, Topological index. A newly proposed quantity characterizing the topological nature of structural isomers of saturated Hydrocarbons, Bull. Chem. soc. Jpn., 44:2332-2339, 1971.

[11] H. Hua, Minimizing a class of unicyclic graphs by means of Hosoya index, Math. Comput. Modelling, 48(5-6):940-948, 2008.

[12] S. Li, X. Li and W. Jing, On the extremal Merrifield-Simmons index and Hosoya index of quasi-tree graphs, Discrete Appl. Math., 157(13):2877-2885, 2009.

[13] S. Li and Z. Zhu, Sharp Lower Bound for the Total Number of Matchings of Tricyclic Graphs, The electronic journal of combinatorics, 17 \#R132, (2010)

[14] S. Li and Z. Zhu, The number of independent sets in unicyclic graphs with a given diameter, Discrete Appl. Math., 157(7):1387-1395, 2009.

[15] S. B. Lin and C. Lin, Trees and forests with large and small independent indices, Chinese J. Math., 23(3):199-210, 1995.

[16] H. Liu, X. Yan and Z. Yan, On the Merrifield-Simmons indices and Hosoya indices of trees with a prescribed diameter, MATCH Commun. Math. Comput. Chem., 57(2):371-384, 2007.

[17] R.E. Merrifield and H.E. Simmons, Topological Methods in chemistry, Wiley, New York, 1989.

[18] J. Ou, On extermal unicyclic molecular graphs with maximal Hosoya index, Discrete Appl. Math., 157(2):391-397, 2009.

[19] X.-F. Pan, J.-M. Xu, C. Yang and M.-J. Zhou, Some graphs with minimum Hosoya index and maximum Merrifield-Simmons index, MATCH Commun. Math. Comput. Chem., 57(1):235-242, 2007.

[20] A. S. Pedersen and P. D. Vestergaard, Bounds on the number of vertex independent sets in a graph, Taiwanese J. Math., 10(6):1575-1587, 2006.

[21] S. Wagner, Extremal trees with respect to Hosoya index and Merrifield-Simmons index, MATCH Commun. Math. Comput. Chem., 57(1):221-233, 2007.

[22] S. G. Wagner and I. Gutman, Maxima and minima of the Hosoya index and the Merrifield-Simmons index: A survey of results and techniques, Acta Applicandae Mathematicae, 112:323-346, 2010.

[23] Y. Ye, X.-F. Pan and H. Liu, Ordering unicyclic graphs with respect to Hosoya indices and Merrifield-Simmons indices, MATCH Commun. Math. Comput. Chem., 59(1):191-202, 2008.

[24] A. Yu and X. Lv, The Merrifield-Simmons indices and Hosoya indices of trees with k pendant vertices, J. Math. Chem., 41(1):33-43, 2007. 


\section{Corrigendum (17 June 2015)}

The authors would like to point out the following two corrections.

Firstly, the formulas in the Abstract and in the last paragraph of Page 2 should be replaced by

$$
91 f_{n-7}+16 f_{n-9} \text { for } n \geqslant 14 \text {. }
$$

Secondly, Theorem 12 should be replaced by the corrected version below.

Theorem 12. Let $G$ be an arbitrary $n$-vertex connected tricyclic graph. If $n=4$ then $z(G) \leqslant 10$ with equality holding if and only if $G \cong H_{4}$.

If $n=5$ then $z(G) \leqslant 16$ with equality holding if and only if $G \cong H_{5}$.

If $n=6$ then $z(G) \leqslant 26$ with equality holding if and only if $G \cong H_{6}$ or $G \cong H_{6}^{\prime}$.

If $n=7$ then $z(G) \leqslant 42$ with equality holding if and only if $G \cong H_{7}$ or $G \cong H_{7}^{\prime}$ or $G \cong H_{7}^{\prime \prime}$ or $G \cong H_{7}^{\prime \prime \prime}$.

If $8 \leqslant n \leqslant 11$ then $z(G) \leqslant 13 f_{n-4}+16 f_{n-5}$ with equality holding if and only if $G \cong H_{n}$. If $n=13$ then $z(G) \leqslant 784$ with equality holding if and only if $G \cong H_{13}$.

If $n=12$ or $n \geqslant 14$ then $z(G) \leqslant 91 f_{n-7}+16 f_{n-9}$ with equality holding if and only if $G \cong H_{n}$. See Figure 10 .

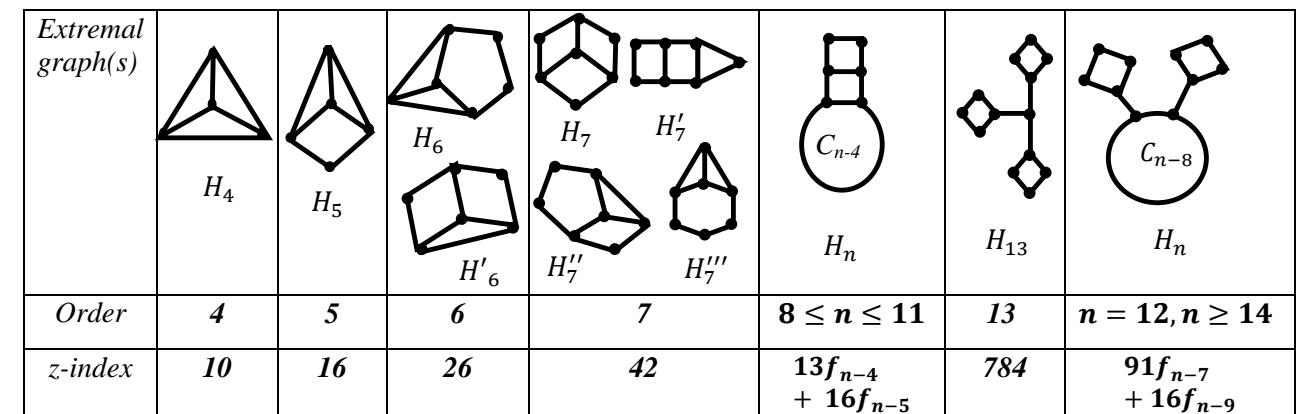

Figure 10: Extremal graphs 